\title{
Subcortical Afferents to the Nucleus Reticularis Tegmenti Pontis in the Rabbit: A Retrograde Horseradish Peroxidase Study
}

\author{
By \\ Tetsu HAYAKAWA and Katuya ZYO \\ Department of Anatomy, Hyogo College of Medicine \\ Mukogawa-cho. Nishinomiya. Hyogo 663. Japan
}

-Received for Publication. June 25. 1986 -

Key Words: Nucleus reticularis tegmenti pontis, Medial mammillary nucleus, Nucleus of the fields of Forel. Tectal projections. Fiber connection

Summary: The subcortical afferent projections to the nucleus reticularis tegmenti pontis (RT) were identified from the retrograde transport of horseradish peroxidase (HRP). HRP was injected into the medial and lateral parts of the RT, respectively. The major afferent inputs to the RT were found to arise ipsilaterally from the nucleus of the fields of Forel, the medial division of the medial mammillary nucleus, the region lateral to the red nucleus, and the posterior commissural nucleus; contralaterally from the anterior pretectal nucleus, the deep layer of the superior colliculus, and the supragenual nucleus; and bilaterally from the lateral habenular nucleus, the retrorubral nucleus, the central and posterior nuclei of the interpeduncular complex, the gray matter along the floor of the fourth ventricle, and the prepositus hypoglossi nucleus. Regions containing fewer labeled cells included the premammillary nucleus, the zona incerta, the nucleus pontis centralis oralis and the medial vestibular nucleus. In addition, the medial division of the medial mammillary nucleus, the lateral habenular nucleus, the central and posterior nuclei of the interpeduncular complex, and the supragenual nucleus were found to project mainly to the medial part of the RT. The superior colliculus, the retrorubral nucleus and the gray matter along the floor of the fourth ventricle projected mainly to the lateral part of the RT.

These results indicate that the medial part of the RT receives fibers mainly from the diencephalic limbic areas, whereas the lateral part of the RT receives fibers mainly from nonlimbic (somatomotor, visuomotor and vestibular) areas.

The nucleus reticularis tegmenti pontis (RT) of the rabbit is situated lateral to the central superior nucleus, dorsal to the pontine gray proper, and ventromedial to the nucleus pontis centralis oralis. It is well known that the RT sends fibers to the cerebellar cortex and deep cerebellar nuclei (Chan-Palay, 1977; Brodal, 1980b, 1982; Blanks et al., 1983), and also receives fibers from the sensori- motor cerebral cortical areas (Brodal and Brodal, 1971). The topographical organization of the cortico-ponto-cerebellar projections has thus been recognized (Brodal, 1980a).

On the other hand, recent anatomical studies employing anterograde autoradiographic methods, have suggested that subcortical afferent projections to the RT

Address for correspondence: Tetsu HAYAKAWA, Department of Anatomy, Hyogo College of Medicine. Mukogawa-cho, Nishinomiya, Hyogo, 663, Japan. Tel. 0798-45-6484. 
arise from the mammillary nuclei (Cruce, 1977, Veazey et al., 1982), the habenular nuclei (Herkenham and Nauta, 1977), the superior colliculus (Holstege and Collewijn, 1982), the pretectal nuclei (Berman, 1977), the zonta incerta (Ricardo, 1981), and the vestibular nuclei (Gerrits et al., 1985). However, there are few comprehensive studies on the afferent projections to the RT, and some discrepancies exist among different authors concerning the details of these connections. Such discrepancies appear to have arisen partly because the habenular, tectal and vestibular inputs project not only to the RT but also to the central superior nucleus (Aghajanian and Wang, 1977; Zyo et al., 1978; Hayakawa and Zyo, 1982; Holstege and Collewijn, 1982) and the nucleus pontis centralis oralis (Kawamura et al., 1974; Graham, 1977; Gerrits et al., 1985), and partly because the degeneration method cannot avoid involvement of fibers-of-passage.

The present study was carried out in order to investigate the original subcortical nuclei projecting to the RT using a retrograde horseradish peroxidase (HRP) method, and to determine whether the projections to the RT are topographically organized.

\section{Materials and Methods}

In total, 28 adult female rabbits weighing 2.0-2.5 kg were used in this study. All surgical procedures were carried out under pentobarbital $(30 \mathrm{mg} / \mathrm{kg}$, i.v.) anesthesia.

The animals were placed in a Kopf stereotaxic apparatus and HRP conjugated with wheat-germ-agglutinin (WGA-HRP) or nonconjugated HRP (Sigma, Type VI) was injected into the pontine tegmental region by microinjection or microiontophoresis (Gonatas et al., 1979). For the microinjection, $20-40 \mathrm{nl}$ of $30 \% \mathrm{HRP}$ or $2 \%$ WGA-HRP (in 0.1 $\mathrm{M}$ Tris buffer, $\mathrm{pH}$ 8.6) was introduced through a $1 \mu$ l Hamilton syringe over a period of $10 \mathrm{~min}$. For the iontophoretic injection, a fine tip glass pipet (O.D. 10-50 $\mu \mathrm{m}$ ) filled with 10\% HRP was inserted, and the HRP was injected with a current of $2-4 \mu \mathrm{A}$ applied in an alternating mode ( $5 \mathrm{~s}$ on $/ 5 \mathrm{~s}$ off) for 4-6 min according to the procedure of Graybiel and Devor (1974). The pipet was kept in situ for an additional $20 \mathrm{~min}$ after the injection in order to restrict the injection site.

Following 20-48 hours survival, the animals were perfused with $0.9 \% \mathrm{NaCl}$ followed by $1.25 \%$ glutaraldehyde- $1 \%$ paraformalde-

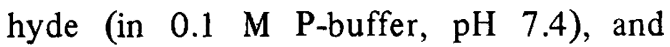
finally with cold $10 \%$ buffered sucrose solution. The brains were removed and stored in phosphate buffer with $10 \%$ sucrose at $4^{\circ} \mathrm{C}$ until sectioning. Serial $40 \mu \mathrm{m}$-thick frozen frontal sections were cut and every third section was reacted with tetramethyl benzidine (TMB) according to the method of Mesulam (1978). All reacted sections were counterstained with neutral red, and HRPlabeled cells were observed under brightfield or darkfield illumination. For cytoarchitectonic studies, serial $15 \mu \mathrm{m}$-thick frontal or parasagittal sections of the formalin-fixed brain were stained by cresyl violet or Klüver-Barrera's method. The cytoarchitectural subdivisions and nomenclature of the rabbit brain were based primarily on the descriptions of Winkler and Potter (1911), Berman (1977) and Gerhard and Olszewski (1969).

\section{Results}

Control injections

HRP or WGA-HRP was injected into the central superior nucleus (cases G209 and G235), and the nucleus pontis centralis oralis (cases G225 and G230) as control experiments.

In the two cases of HRP injection into the central superior nucleus, the HRP de- 


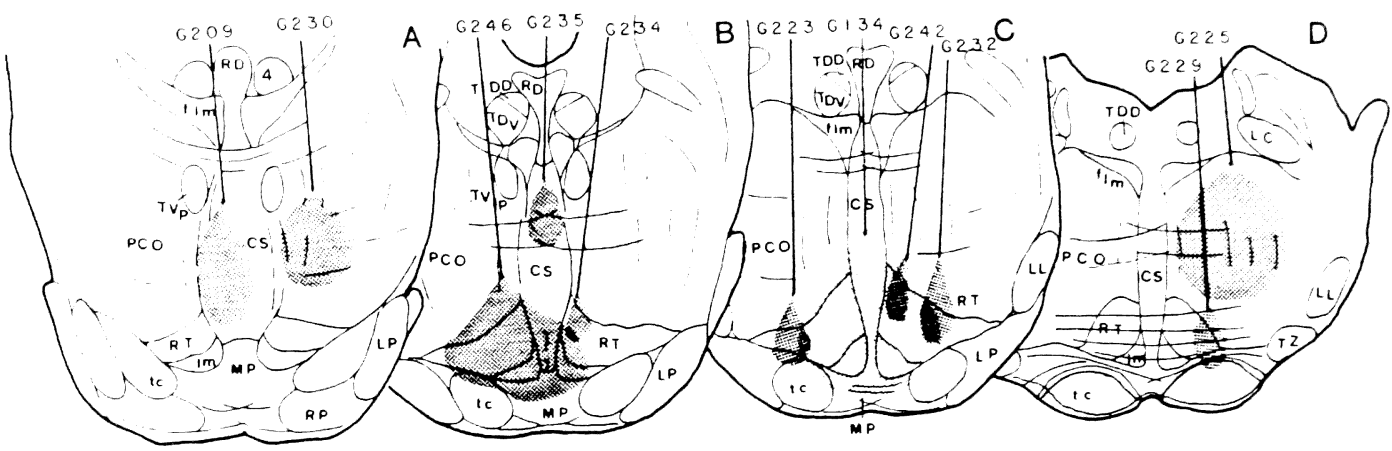

Fig. 1. Schematic diagrams of frontal sections showing the central parts of the injection sites in different regions of the pontine tegmental area. Shaded areas indicate injection sites, and thick lines indicate pipet tracks.

posits were found to be restricted within the ventral part (G209) or the dorsal part (G235) of the central superior nucleus (Figs. 1 A and B). In these cases, many HRPlabeled cells were present bilaterally in the lateral habenular nucleus and the medial habenular nucleus, as well as several cells in the paraventricular nucleus of the thalamus (Fig. 2C). The labeled cells in the medial habenular nucleus were lay along the borders of the lateral habenular nucleus (Fig. 8 ). There were many labeled cells in the posterior nucleus of the interpeduncular complex (IP), and several labeled cells in the central and paramedian nuclei of the IP (Figs. 2D and E). Several labeled cells were also found in the nucleus of the diagonal band of Broca, the lateral hypothalamic nucleus, the dorsal raphe nucleus, the laterodorsal tegmental nucleus, the nucleus pontis centralis oralis, the gray matter along the floor of the fourth ventricle, and the medial vestibular nucleus (Figs. 2A-I). A few labeled cells were present in the preoptic area and the retrorubral nucleus, but no labeled cells were observed in the mammillary, the subthalamic, the pretectal, or the perihypoglossal region (Table 1).

Slightly large WGA-HRP injections were made into the nucleus pontis centralis oralis in cases G225 and G230. The center of the WGA-HRP deposits was more rostromedial in case G230 (Fig. 1A) than in case G225 (Fig. 1D). The distribution of WGA-HRP deposits in both cases was extended within the nucleus pontis centralis oralis. In case G225, many WGA-HRP-labeled cells were found ipsilaterally in the nucleus of the fields of Forel, contralaterally in the anterior pretectal nucleus, the lateral onethird of the deep layer of the superior colliculus, and the nucleus pontis centralis oralis; and bilaterally in the prepositus hypoglossi nucleus (Table 1). A few labeled cells were present in the ipsilateral nucleus of the posterior commissure, while sporadic but consistent labeled cells were observed ipsilaterally in the interstitial nucleus of Cajal, the nucleus of Darkschewitsch, and the retrorubral nucleus, and bilaterally in the medial vestibular nucleus. In case G230, there were also labeled cells in the bilateral midbrain periaqueductal gray matter, and just a few in the lateral hypothalamic nucleus, and the lateral habenular nucleus ipsilaterally (Table 1).

\section{Injections into the RT}

In the experiments involving large WGAHRP injections, the WGA-HRP deposits were centered in the ventral part of the 


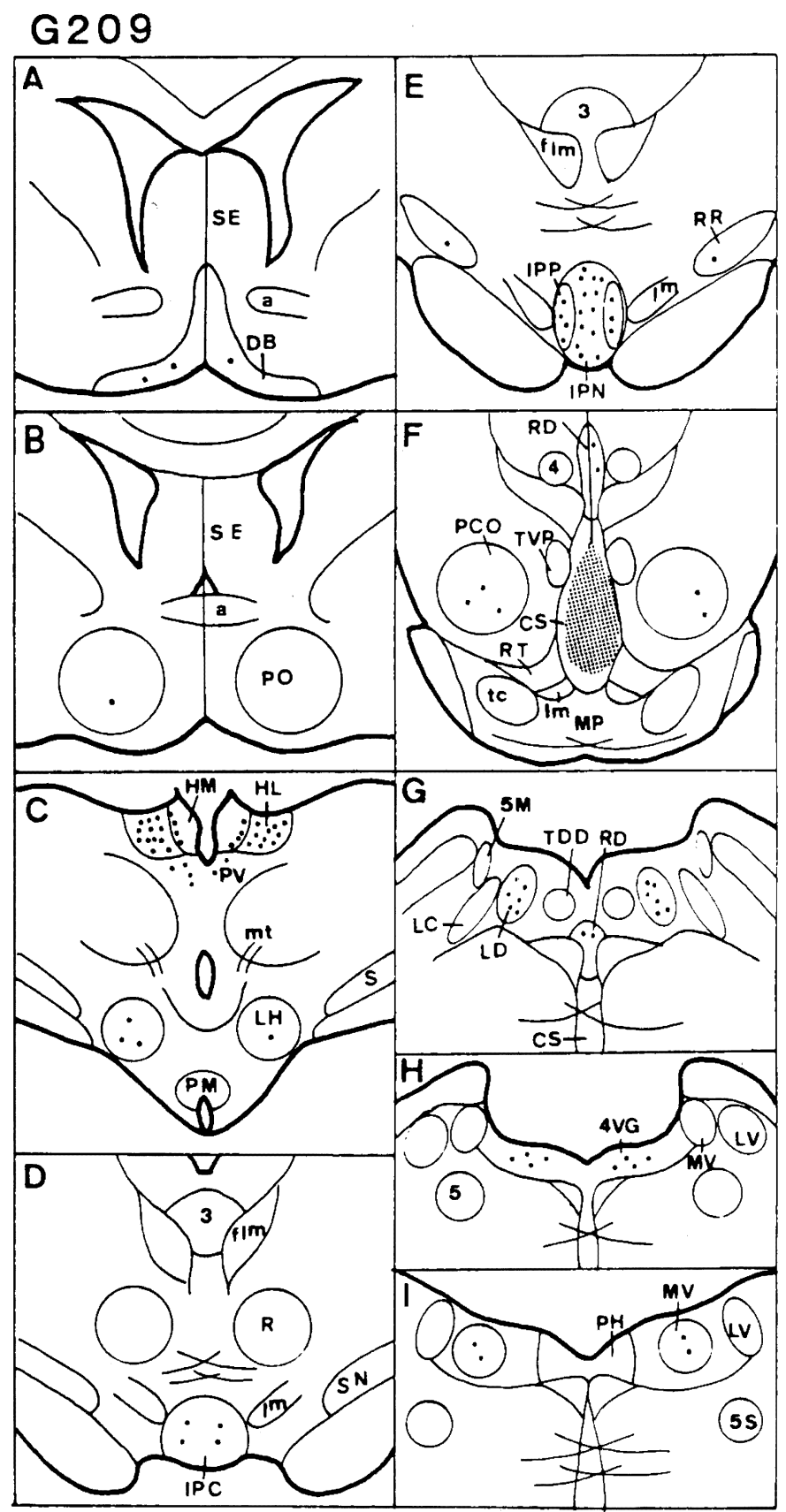

Fig. 2. Schematic drawings of the distribution of HRP-labeled cells after injection into the central superior nucleus in case G209. The shaded area indicates the HRP injection site, and each dot indicates a few labeled cells. 
Table 1. Distribution of HRP-labeled Cells.

The densities of labeled cells were as follows: $+=0-1$ cell per section; $++=2-5$ cells per section; $+++6-10$ cells per section; $+++=$ more than 10 cells per section.

\begin{tabular}{|c|c|c|c|c|c|c|c|c|c|c|c|}
\hline \multirow{3}{*}{ Area } & \multicolumn{11}{|c|}{ Injection Sites } \\
\hline & \multicolumn{7}{|c|}{ Nucleus reticularis tegmenti pontis } & \multicolumn{4}{|c|}{ Control injection } \\
\hline & G134 & G246 & G234 & G242 & $\mathrm{G} 232$ & G223 & G229 & G209 & G235 & G225 & $\mathrm{G} 230$ \\
\hline N. diagonal band of Broca & $a++$ & + & & & & & & ++ & + & & \\
\hline Preoptic area & ++ & ++ & & & & & & + & & & \\
\hline Lateral hy pothalamic $n$. & ++++ & +++ & & & + & + & + & ++ & & & + \\
\hline Premammillary $n$. & ++ & + & & & + & + & & & & & \\
\hline Medial mammillary $n$. & ++++ & +++ & +++ & ++ & & & & & & & \\
\hline Lateral mammillary $\mathrm{n}$. & ++ & + & & & & & & & & & \\
\hline Medial habenular $\mathrm{n}$. & +++ & + & & & & & & +++ & ++ & & \\
\hline Lateral habenular $\mathrm{n}$. & ++++ & +++ & +++ & +++ & & & & ++++ & ++++ & & + \\
\hline $\begin{array}{l}\text { Paraventricular } \mathrm{n} \text {. } \\
\text { of thalamus }\end{array}$ & ++ & ++ & & & & & & ++ & ++ & & \\
\hline N. of fields of Forel & ++++ & ++++ & ++ & ++ & ++++ & +++ & ++++ & & & +++ & ++ \\
\hline Zona incerta & ++ & +++ & + & & + & & & & & & \\
\hline $\begin{array}{l}\text { Region lateral to } \\
\text { red nucleus }\end{array}$ & ++ & +++ & ++ & & +++ & & ++ & & & & + \\
\hline $\begin{array}{l}\text { Midbrain periaque- } \\
\text { ductal gray matter }\end{array}$ & +++ & ++ & & & & & + & & & & ++ \\
\hline
\end{tabular}

\begin{tabular}{|c|c|c|c|c|c|c|c|c|c|c|c|}
\hline $\begin{array}{l}\text { Posterior commissural } \\
\text { n. }\end{array}$ & + & +++ & + & + & ++ & ++ & ++ & & & ++ & \\
\hline Anterior pretectal $\mathrm{n}$. & ++ & +++ & ++ & ++ & +++ & ++ & ++ & & & ++ & +++ \\
\hline Superior colliculus & ++ & ++ & + & & ++ & + & + & & & +++ & ++ \\
\hline $\begin{array}{l}\text { Interstitial n. of } \\
\text { Cajal }\end{array}$ & ++ & + & & & & & & & & + & + \\
\hline N. Darkschewitsch & ++ & + & & & & & + & & & + & + \\
\hline Central n. of IP & +++ & ++ & ++ & + & & & & ++ & + & & \\
\hline Paramedian $n$. of IP & +++ & ++ & & & & & & ++ & ++ & & \\
\hline Posterior n. of IP & ++ & ++ & ++ & + & & & & ++ & ++ & & \\
\hline Retrorubral n. & ++ & +++ & & & +++ & + & +++ & + & & + & ++ \\
\hline $\begin{array}{l}\mathrm{N} \text {. pontis centralis } \\
\text { oralis }\end{array}$ & +++ & +++ & & & + & + & & ++ & + & +++ & ++ \\
\hline
\end{tabular}

N. raphe dorsalis

Laterodorsal tegmental n.

Gray matter along floor of fourth ventricle

Medial vestibular $n$.

Supragenual $\mathrm{n}$.

Prepositus hypoglossi n.

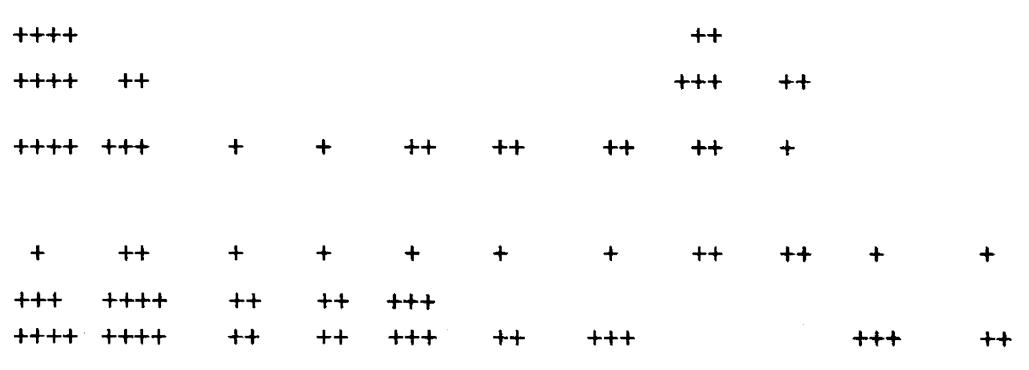


central superior nucleus (G134) (Figs. 1C and $5 \mathrm{~A})$ or the ventromedial part of the RT (G246) (Fig. 1B). In both cases, the WGA-HRP deposits extended bilaterally into the ventral part of the central superior nucleus, the RT, the lemniscus medialis, the dorsal part of the medial pontine nucleus, and the ventromedial part of the nucleus pontis centralis oralis. Numerous WGA-HRPlabeled neurons were found bilaterally in the medial mammillary nucleus (Figs. 6 and 7), and a few labeled cells were present in the lateral mammillary nucleus and the premammillary nucleus (Table 1). According to Bleier's (1969) nomenclature, the medial mammillary nucleus of the rabbit is divided into medial, intermediate, basal, and posterior divisions. In this case, many labeled cells were found only in the bilateral medial divisions, and they formed a V-shape (Fig. 6). There were many labeled cells in the lateral habenular nucleus, the lateral part of the medial habenular nucleus, the nucleus of the fields of Forel (Fig. 9), and the zona incerta. In the medial habenular nucleus, many labeled cells were detected in case G134, but only a few were found in case G246 (Table 1). There were also labeled cells in the nucleus of the diagonal band of Broca, the preoptic area, the lateral hypothalamic nucleus, and the nucleus paraventricularis thalami.

In the midbrain, many labeled cells occurred in the nucleus of the posterior commissure, the anterior pretectal nucleus, the superior colliculus, mainly the lateral one-third of the deep layer, the region lateral to the red nucleus, the rostral part of the midbrain periaqueductal gray matter, and the central, paramedian and posterior nuclei of the IP, but no labeled cells were found in the nucleus of the optic tract. A few labeled cells were found in the interstitial nucleus of Cajal and the nucleus of Darkschewitsch. There were also many labeled cells in the prepositus hypoglossi nucleus, the supragenual nucleus, the gray matter along the floor of the fourth ventricle, and the laterodorsal tegmental nucleus, several cells in the retrorubral nucleus, and the nucleus pontis centralis oralis; and just a few cells in the medial vestibular nucleus. In the nucleus raphe dorsalis, many labeled cells were present in case G134 but no labeled cells were observed in case G246 (Table 1).

Restricted HRP injections were made into the medial part of the RT in cases G234 and G242. The HRP deposits were found to be restricted within the unilateral mediodorsal part of the RT, and did not extend to the central superior nucleus (Figs. 1B, C and 5B). In case G234 (Fig. 3), many HRP-labeled cells were observed ipsilaterally in the medial division of the medial mammillary nucleus, and the nucleus of the fields of Forel; and bilaterally in the lateral habenular nucleus (Figs. 3A and B). Several labeled cells were detected in the central and posterior nuclei of the IP (Figs. 3D and 10), the ipsilateral region lateral to the red nucleus, the contralateral anterior pretectal nucleus (Fig. 3C), the contralateral supragenual nucleus, and the bilateral prepositus hypoglossi nucleus (Figs. 3F and G). There were also a few labeled cells ipsilaterally in the zona incerta, and the nucleus of the posterior commissure, contralaterally in the deep layer of the superior colliculus, and bilaterally in the gray matter along the floor of the fourth ventricle, and the medial vestibular nucleus. In case G242, no labeled cells were found in the zona increta and the region lateral to the red nucleus (Table 1).

Experiments in which the HRP deposits involved the lateral part of the RT and the ventral edge of the nucleus pontis centralis oralis included case G232, G223 and G229 (Figs. 1C, D and 5C). In case G232 (Fig. 4), many HRP-labeled cells were found ipsilaterally in the nucleus of the fields of Forel, and the region lateral to the red nucleus; contralaterally in the anterior pretectal nucleus 
G234

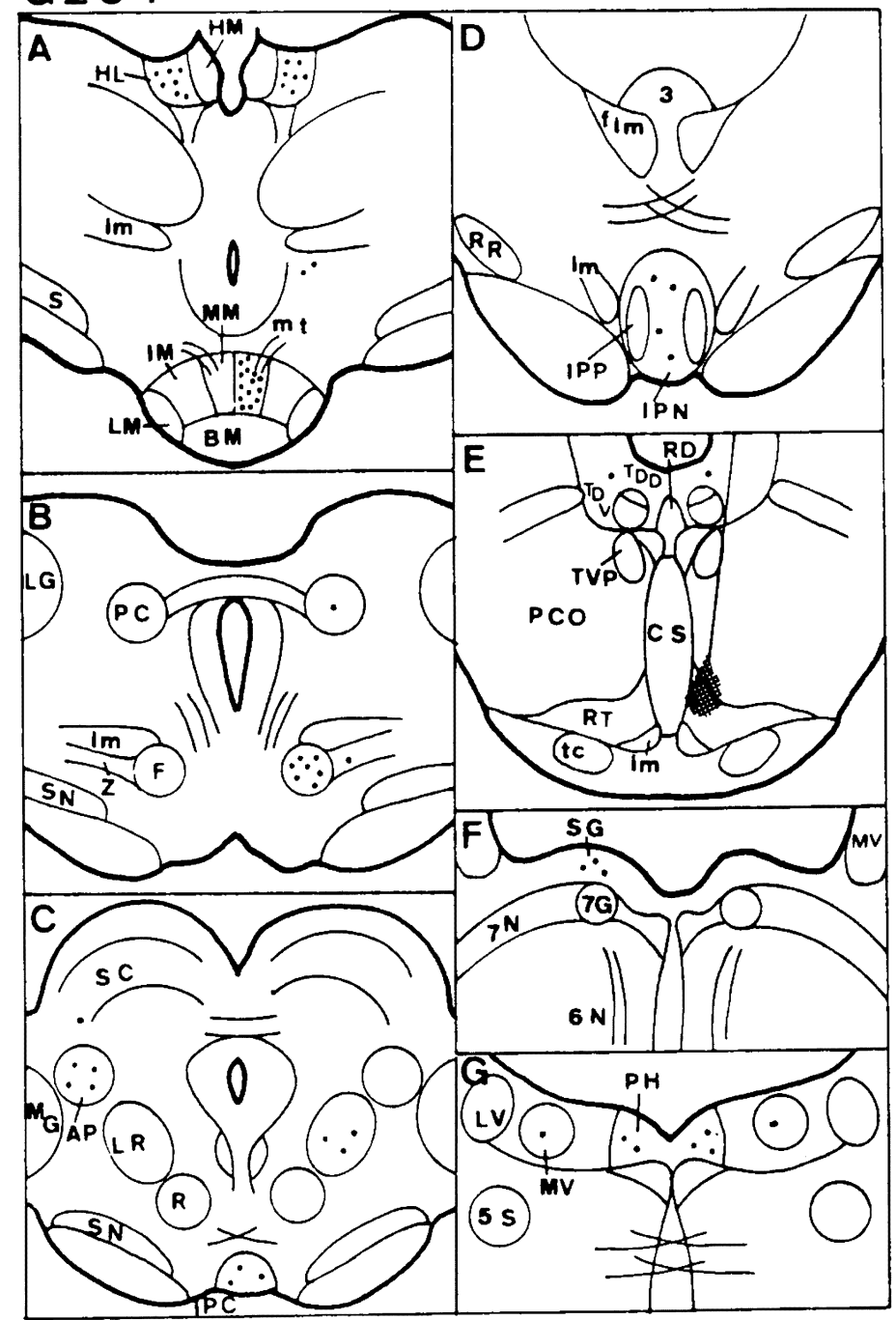

Fig. 3. Schematic drawings of the distribution of HRP-labeled cells after injection into the medial part of the nucleus reticularis tegmenti pontis (RT) in case G234. The shaded area indicates the HRP injection site, and each dot indicates a few labeled cells.

(Figs. 4B-D), and the superagenual nucleus (Fig. 13); and bilaterally in the retrorubral nucleus (Fig. 11) and the prepositus hypoglossi nucleus (Fig. 12). Several labeled cells were present in the ipsilateral nucleus of the posterior commissure, the contralateral deep layer of the superior colliculus (Figs. $4 C$ and $D$ ), and the bilateral gray matter along the floor of the fourth ventricle (Fig.
4H). Only a few but consistently labeled cells were detected in the lateral hypothalamic nucleus, the premammillary nucleus and the medial vestibular nucleus ipsilaterally. No labeled cells were found in the mammillary nuclei, the habenula and the IP. In cases G223 and G229, the HRP deposits in the RT were centered more laterally than those of G232. The labeling patterns were 


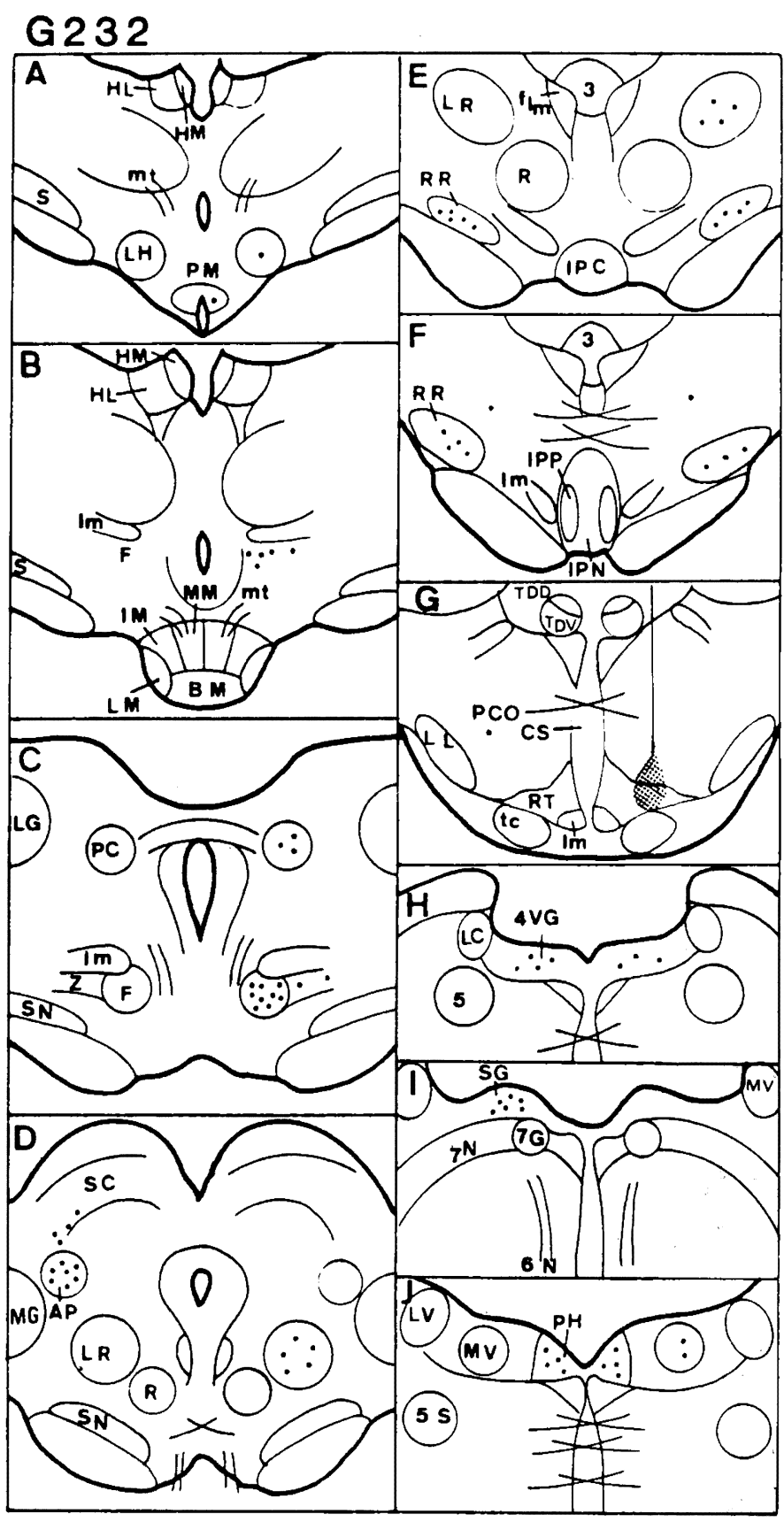

Fig. 4. Schematic drawings of the distribution of HRP-labeled cells after injection into the lateral part of the RT in case G232. The shaded area indicates the HRP injection site, and each dot indicates a few labeled cells. 
almost the same in these cases, but no labeled cells were observed in the zona incerta and the supragenual nucleus (Table 1).

\section{Discussion}

The present results demonstrate that the $\mathrm{RT}$ receives its main inputs ipsilaterally from the medial division of the medial mammillary nucleus, the nucleus of the fields of Forel, the region lateral to the red nucleus, and the posterior commissural nucleus; contralaterally from the anterior pretectal nucleus, the deep layer of the superior colliculus, and the supragenual nucleus; and bilaterally from the lateral habenular nucleus, the central and posterior nuclei of the IP, the gray matter along the floor of the fourth ventricle, and the prepositus hypoglossi nucleus.

Although uninjured axons of fibers-ofpassage may not appear to take up and transport free HRP (FitzGibbon et al., 1983), some problems do arise in the interpretation of the present findings for HRP rabeled cells (Herkenham and Nauta, 1977). Brodal et al. (1983) have reported that the number of retrogradely WGA-HRP labeled cells taken up from the fibers-of-passage was much smaller than that after similar free HRP injections. When large WGA-HRP injections were made into the RT in the present study, the injection sites were extended to the fibers of the lemniscus medialis. In these cases, there were no labeled cells in the gracile or cunate nuclei. In addition, when WGA-HRP cases were compared with HRP ones, the overall distribution of retrograde cell labeling was found to be similar (Table 1). Therefore, uptake of HRP from fibersof-passage may not contribute significantly to the overall afferent pattern of the RT.

When large WGA-HRP injections were made into the RT, labeled cells were found in the nucleus of the diagonal band of Broca, the preoptic area, the lateral hypothalamic nucleus, the medial habenular nucleus, the paraventricular nucleus of the thalamus, the paramedian nucleus of the IP, the dorsal raphe nucleus and the laterodorsal tegmental nucleus. However, all these nuclei were labeled in the case of either a large injection including the central superior nucleus or restricted injections into the central superior nucleus, but no labeled cells were observed in the case of restricted injections into the RT. These nuclei are therefore considered to project not to the RT but to the central superior nucleus.

Many authors have reported that the lateral habenular nucleus projects to the pontine tegmentum in the rat (Herkenham and Nauta, 1979), cat (Akagi and Powell, 1968; Zyo et al., 1978), rabbit (Cragg, 1961), opossum (Way and Kaelber, 1969; Smaha and Kaelber, 1973), and wallaby (Way, 1975). The present findings also indicate that the medial part of the RT and the central superior nucleus receive fibers from the lateral habenular nucleus, but neither the lateral part of the RT nor the nucleus pontis centralis oralis receive lateral habenular inputs. The lateral habenular nucleus thus projects to the paramedian pontine tegmental region including the medial pontine nucleus, the medial part of the RT, the central superior nucleus, the ventral and the dorsal tegmental nuclei of Gudden, the dorsal raphe nucleus, the laterodorsal tegmental nucleus, and the nucleus of the locus caeruleus.

The descending fibers from the medial habenular nucleus terminate at the IP in a topographical manner in the rat (Marchand et al., 1980; Contestabile and Flumerfelt, 1981) and the cat (Hayakawa and Zyo, 1982). In the present study, neurons in the lateral border of the medial habenular nucleus were also labeled after HRP injection into the central superior nucleus. Herkenham and Nauta (1979), in their an- 
terograde autoradiographic tracing study in the rat, reported sparse projections from the medial habenular nucleus not to the RT but to the central superior nucleus. Descending projections from each of the subnuclei of the IP to the central superior nucleus and Gudden's tegmental nuclei have been reported in the rat (Liu et al., 1984; Shibata and Suzuki, 1984), rabbit (Hayakawa and Zyo, 1985) and cat (Smaha and Kaelber, 1973; Hayakawa et al., 1981). Our experiments also revealed that the medial part of the RT receives fibers from the central and posterior nuclei of the IP in the rabbit.

Our interesting finding in the present study was that many labeled cells were found only in the medial division of the ipsilateral medial mammillary nucleus after HRP or WGA-HRP injection into the medial part of the RT. Degenerating and autoradiographic tracing studies have demonstrated that the mammillary nuclei project to the pontine gray proper and the RT in the rat (Cruce, 1977), cat (Niimi et al., 1972), rabbit (Ban and Zyo, 1963), and cynomolgus monkey (Veazey et al., 1982). These anterograde tracing studies did not show subnuclei of the medial mammillary nucleus projecting to the pontine tegmentum. When large WGA-HRP injections were made by us into the RT and the medial pontine nucleus, WGA-HRP-labeled cells were present in the lateral mammillary nucleus in cases G134 and G246. This suggests that the lateral mammillary nucleus projects not to the RT but to the medial pontine nucleus. Our previous experiments (Hayakawa and Zyo, 1984, 1985) have demonstrated that the medial division of the medial mammillary nucleus sends fibers to and receives fibers from the pars principalis of the ventral tegmental nucleus of Gudden, while the lateral mammillary nucleus sends fibers to and receives fibers from the dorsal tegmental nucleus of Gudden, ipsilaterally. Van der Kooy et al. (1978) reported in the rat that the neurons of the medial part of the medial mammillary nucleus send fibers both to the ipsilateral anteroventral nucleus of the thalamus and into the mammillo-tegmental tract. The neurons projecting into the mamillo-tegmental tract may thus send off collateral fibers, which terminate not only at the ventral tegmental nucleus of Gudden, but also at the medial part of the RT.

The RT is cytoarchitectonically clearly distinct from the nucleus pontis centralis oralis, but both the RT and the nucleus pontis centralis oralis receive many fibers from the nucleus of the fields of Forel, the zona incerta, the retrorubral nucleus, and the region lateral to the red nucleus. Many reports have indicated that the midbrain and pontine reticular formation including the nucleus pontis centralis oralis and the RT, receive fibers from the nucleus of the fields of Forel and the zona incerta in the rat (Ricardo, 1981; Watanabe and Kawana, 1982; Shammah-Lagnado et al., 1983), cat (Kaelber and Smith, 1979) and rhesus monkey (Schnyder et al., 1985), but the RT received less fibers from the zona incerta in the present study. The major subcortical afferents to the medullary paramedian reticular nuclei are from the interstitial nucleus of Cajal, the nucleus of Darkschewitsch and the midbrain and pontine reticular formation (Elisevich et al., 1985). The RT receives few fibers from these nuclei, so that the RT may be included not in the medullary but in the pontine reticular core.

Although many authors have demonstrated the pretectal and superior collicular projections to the RT and the pontine reticular formation (Kawamura et al., 1974; Berman, 1977; Graham, 1977; Itoh, 1977; Kawamura and Hshikawa, 1978; Holstege and Collewijn, 1982), some discrepancies do exist concerning the original nuclei of the tecto-reticular projections. This is 
partly because there are many subnuclei in the pretectum and layers in the superior colliculus (Kanaseki and Sprague, 1974), or partly because injection sites of tritiumlabeled amino acids are liable to involve several subnuclei when using the anterograde autoradiographic tracing method. The present findings demonstrated that the anterior pretectal nucleus and the deep layer of the superior colliculus project contralaterally to the RT, while the nucleus of the posterior commissure projects ipsilaterally to the RT. On the other hand, recent electrophysiological studies have indicated that the RT neurons were antidromically activated from the flocculus and orthodromically activated from the optic tracts, so that the RT neurons could transfer visual signals to the flocculus (Maekawa et al., 1981; Miyashita and Nagao, 1984). However, the RT was not found to receive direct projections from the nucleus of the optic tract in the present experiments or in other autoradiographic anatomical studies (Berman, 1977; Itoh, 1977; Holstege and Collewijn, 1982). The RT also has connections with the prepositus hypoglossi nucleus and the supragenual nucleus as well as the flocculus (Brodal, A., 1952; Brodal, P., 1980b, 1982; Blanks et al., 1983), and less connections with the medial vestibular nucleus (Balaban, 1983; Gerritis, 1983). These results suggest that the RT, especially the medial part of the RT, may contribute to optokinetic movements through the cerebellar and vestibular nuclei as well as the diencephalic limbic nuclei.

\section{Acknowledgements}

The authors gratefully thank Drs. M. Seki and K. Kurohmaru for their helpful discussions, and Miss M. Okayama and Miss F. Karitani for the technical assistance.

\section{References}

1) Aghajanian, G.K. and Wang, R.Y.: Habenular and other midbrain raphe afferents demonstrated by a modified retrograde tracing technique. Brain Res., 122: 229-242, 1977.

2) Akagi, K. and Powell, E.W.: Differential projections of habenular nuclei. J. Comp. Neurol., 132: 263-274, 1968.

3) Balaban, C.D.: A projection from nucleus reticularis tegmenti pontis of Bechterew to the medial vestibular nucleus in rabbits. Exp. Brain Res., 51 : 304-309, 1983.

4) Ban, T. and Zyo, K.: Experimental studies on the mammillary peduncle and mammillotegmental tracts in the rabbit. Med. J. Osaka Univ., 13: 242-270, 1963.

5) Berman, A.L.: The Brain Stem of the Cat. Wisconsin Univ. Press, Madison, 1968.

6) Berman, N.: Connections of the pretectum in the cat. J. Comp. Neurol., 174: 227-257, 1977.

7) Blanks, R.H.I., Precht, W. and Torigoe, Y.: Afferent projections to the cerebellar flocculus in the pigmented rat demonstrated by retrograde transport of horseradish peroxidase. Exp. Brain Res., 52: 293-306, 1983.

8) Bleier, R.: Retrograde transsynaptic cellular degeneration in mammillary and ventral tegmental nuclei following limbic decortication in rabbits of various ages. Brain Res., 12: 365-393, 1969.

9) Brodal, A.: Experimental demonstration of cerebellar connexions from the peri-hypoglossal nuclei (nucleus intercalatus, nucleus praepositus hypoglossi and nucleus of Roller) in the cat. J. Anat., 86: 110-129, 1952.

10) Brodal, A. and Brodal, P.: The organization of the nucleus reticularis tegmenti pontis in the cat in the light of the experimental anatomical studies of its cerebral cortical afferents. Exp. Brain Res., 13: 90-110, 1971.

11) Brodal, P.: The cortical projection to the nucleus reticularis tegmenti pontis in the rhesus monkey. Exp. Brain Res., 38: 19-27, 1980a.

12) Brodal, P.: The projection from the nucleus reticularis tegmenti pontis to the cerebellum in the rhesus moneky. Exp. Brain Res., 38: 2936, $1980 \mathrm{~b}$.

13) Brodal, P.: Further observations on the cerebellar projections from the pontine nuclei and the nucleus reticularis tegmenti pontis in the rhesus moneky. J. Comp. Neurol., 204 : 
44-55, 1982.

14) Brodal, P., Dietrichs, E., Bjaalie, J.G., Nordby, T. and Walberg. F.: Is lectin-coupled horseradish peroxidase taken up and transported by undamaged as well as by damaged fibers in the central nervous system? Brain Res., 278: $1-9,1983$.

15) Chan-Palay, V.: Cerebellar Dentate Nucleus: Organization, Cytology and Transmitters. pp. 275-363, Springer-Verlag, Berlin, 1977.

16) Contestabile, A. and Flumerfelt, B.A.: Afferent connections of the interpeduncular nculeus and the topographic organization of the habenulo-interpeduncular pathway: An HRP study in the rat. J. Comp. Neurol., 196: 253-270, 1981.

17) Cragg, B.G.: The connections of the habenula in the rabbit. Exp. Neurol., 3: 388-409, 1961.

18) Cruce, J.A.: An autoradiographic study of the descending connections of the mammillary nuclei of the rat. J. Comp. Neurol., 176: 631644, 1977.

19) Elisevich, K., Hrycyshyn, A.W. and Flumerfelt, B.A.: Supramedullary projections to the dorsal and ventral divisions of the paramedian reticular nucleus in the cat. Exp. Brain Res., 58: 368-378, 1985.

20) FitzGibbon, T., Kerr,L. and Burke, W.: Uptake of horseradish peroxidase by axons of passage and its modification by poly-Lornithine and dimethylsulfoxide. J. Neurosci. Method., 7: 73-88, 1983.

21) Gerhard, L. and Olszewski, J.: Medulla Oblongata and Pons: Primatologia. Vol. II, Teil 2, Lieferung 3 Karger, Basel, 1969.

22) Gerrits, N.M., Voogt, J. and Magras, I.N.: Vestibular nuclear efferents to the nucleus raphe pontis, the nucleus reticularis tegmenti pontis and the nuclei pontis in the cat. Neurosci. Lett., 54: 357-362, 1985.

23) Gonatas, N.K., Harper, C., Mizutani, T. and Gonatas, J.O.: Superior sensitivity of conjugates of horseradish peroxidase with wheat germ agglutinin for studies of retrograde axonal transport. J. Histochem. Cytochem., 27: 728-734, 1979.

24) Graham, J.: An autoradiographic study of the efferent connections of the superior colliculus in the cat. J. Comp. Neurol.,173: 629-654, 1977.

25) Graybiel, A.M. and Devor, M.: A microelectrophoretic delivery technique for use with horseradish peroxidase. Brain Res., 68 : 167-173, 1974.

26) Hayakawa. T. and Zyo, K.: Organization of the habenulo-interpeduncular connections in cats: A horseradish peroxidase study. Brain Res.,240: 3-11, 1982.

27) Hayakawa, T. and Zyo, K.: Comparative anatomical study of the tegmentomammillary projections in some mammals: A horseradish peroxidase study. Brain Res., 300: 35-349. 1984.

28) Hayakawa. T. and Zyo. K.: Afferent connections of Gudden's tegmental nuclei in the rabbit. J. Comp. Neurol., 235: 169-181. 1985.

29) Hayakawa, T., Seki, M. and Zyo, K.: Studies on the efferent projections of the interpeduncular complex in cats. Okajimas Folia Anat.Jpn., 58: 1-16. 1981.

30) Herkenham, M. and Nauta, W.J.H.: Afferent connections of the habenular nuclei in the rat: A horseradish peroxidase study, with a note on the fibers-of-passage problems. J. Comp. Neurol., 173: 123-146, 1977.

31) Herkenhan, M. and Nauta, W.J.H.: Efferent connections of the habenular nuclei in the rat. J. Comp. Neurol., 187: 19-48, 1979.

32) Holstege, G. and Collewijn, H, : The efferent connections of the nucleus of the optic tract and the superior colliculus in the rabbit. J. Comp. Neurol., 209: 139-175. 1982.

33) Itoh, K.. Efferent projections of the pretectum in the cat. Exp. Brain Res.,30: 89$105,1977$.

34) Kaelber, W.W. and Smith. T.B.. Projections of the zona incerta in the cat, with stimulation controls. Exp. Neurol., 63: 177-200, 1979.

35) Kanaseki. T. and Sprague, J.M.: Anatomical organization of pretectal nuclei and tectal laminae in the cat. J. Comp. Neurol., 158: 319-338, 1974.

36) Kawamura, K. and Hashikawa, T.: Cell bodies of origin of reticular projections from the superior colliculus in the cat: An experimental study with the use of horseradish peroxidase as a tracer. J. Comp. Neurol.,182: 1-16, 1978.

37) Kawamura, K., Brodal. A. and Hoddevik,G.: The projection of the superior colliculus onto the reticular formation of the brain stem: An experimental anatomical study in the cat. Exp. Brain Res., 19: 1-19, 1974.

38) Liu, R., Chang, L. and Wickern, G.: The dorsal tegmental nucleus. An axoplasmic transport study. Brain Res., 310 123-132, 1984.

39) Maekawa, K., Takeda. T. and Kimura. M.: Neuronal activity of nucleus reticularis teg- 
menti pontis: The origin of visual mossy fiber afferents to the cerebellar flocculus of rabbits. Brain Res., 210: 17-30, 1981.

40) Marchand, E.R.. Riley, J.N. and Moor, R.Y.: Interpenduncular nucleus afferents in the rat. Brain Res., 193. 339-352. 1980.

41) Mesulam. M.-M.: Tetramethyl benzidine for horseradish peroxidase neurohistochemistry: A non-carcinogenic blue reaction-product with superior sensitivity for visualizing neural afferents and efferents. J. Histochem. Cytochem., 26. 106-117. 1978.

42) Miyashita, Y. and Nagao, S.: Analysis of signal content of Purkinje cell responses to optokinetic stimuli in the rabbit cerebellar flocculus by selective lesions of brain stem pthways. Neurosci. Res., 1: 223-241, 1984.

43) Niimi, K., Koizuka, M., Kawamura, S. and Abe, K.: Efferent projections of the mamillary body in the cat. Okajimas Folia Anat. Jpn., 49: 129-156, 1972.

44) Ricardo, J.A.: Efferent connections of the subthalamic region in the rat: II. The zona incerta. Brain Res., 214: 43-60, 1981.

45) Schnyder, H., Reisine, H., Hepp, K. and Henn, V.: Frontal eye field projection to the paramedian pontine reticular formation traced with wheat germ agglutinin in the monkey. Brain Res., 329: 151-160, 1985.

46) Shammah-Lagnado, S.J., Ricardo, J. A. Sakamoto, N.T.M.N. and Negrão, N.: Afferent connections of the mesencephalic reticular formation: A horseradish peroxidase study in the rat. Neurosci., 9: 391-409, 1983.

47) Shibata. H. and Suzuki. T.: Efferent projections of the interpeduncular complex in the rat, with special reference to its subnuclei: A retrograde horseradish peroxidase study.
Brain Res., 296 : 345-349, 1984.

48) Smaha, L.A. and Kaelber. W.W.: Efferent fiber projections of the habenula and the interpeduncular nucleus: An experimental study in the opossum and cat. Exp. Brain Res., 16: 291-308, 1973.

49) Van der Kooy, D.. Kuypers. H.G.J.M. and Catsman-Berrevoets, C.E.: Single mammillary body cells with divergent axon collaterals: Demonstration by a simple. fluorescent retrograde double labeling technique in the rat. Brain Res., 158: 189-196, 1978.

50) Veazey, R.B., Amaral, D.G. and Cowan, W.M.: The morphology and connections of the posterior hypothalamus in the cynomolgus monkey (Macaca fascicularis): II. Efferent connections. J. Comp. Neurol., 207: 135-156. 1982.

51) Watanabe, K. and Kawana, E.: The cells of origin of the incertofugal projections to the tectum, thalamus, tegmentum and spinal cord in the rat: A study using the autoradiographic and horseradish peroxidase methods. Neurosci., 7: 2389-2406, 1982.

52) Way, J.S.: A degeneration study of some habenular efferents to the midbrain in a wallaby. Am. J Anat., 142: 1-14, 1975.

53) Way. J.S. and Kaelber. W.W.: A degeneration study of efferent connections of the habenular complex in the opossum. Am. J. Anat., 124: 31-46, 1969.

54) Winkler, C. and Potter. A.: An Anatomical Guide to Experimental Researches on to the Rabbit's Brain. Versluys, Amsterdam, 1911.

55) Zyo. K.. Hayakawa, T., Seki, M. and Ryu. T.: Caudal projections of the habenular nuclei in cats: Fibers from the lateral habenular nucleus. Med. J. Osaka Univ., 28 : 229-243. 1978.

\begin{tabular}{|c|c|c|c|}
\hline \multicolumn{4}{|c|}{ Abbreviations } \\
\hline a & : Anterior commissure & HM & : Medial habenular nucleus \\
\hline AP & : Anterior pretectal nucleus & IM & : Intermediate division of medial mammil- \\
\hline BM & $\begin{array}{l}\text { Basal division of medial mammillary } \\
\text { nucleus }\end{array}$ & IP & $\begin{array}{l}\text { lary nucleus } \\
\text { : Interpeduncular complex }\end{array}$ \\
\hline $\mathrm{C}$ & : Interstitial nucleus of Cajal & IPC & : Central nucleus of IP \\
\hline CS & : Central superior nucleus of raphe & IPN & : Posterior nucleus of IP \\
\hline D & : Nucleus of Darkschewitsch & IPP & : Paramedian nucleus of IP \\
\hline DB & : Nucleus of diagonal band of Broca & LC & : Nucleus of locus caeruleus \\
\hline $\mathrm{F}$ & : Nucleus of fields of Forel & LD & : Laterodorsal tegmental nucleus \\
\hline flm & : Fasciculus longitudinalis medialis & LG & : Lateral geniculate body \\
\hline $\mathrm{HL}$ & : Lateral habenular nucleus & LH & : Lateral hypothalamic nucleus \\
\hline
\end{tabular}


LL : Ventral nucleus of lateral lemniscus

LM : Lateral mammillary nucleus

Im : Lemniscus medialis

LP : Lateral pontine nucleus

LR : Region lateral to red nucleus

LV : Lateral vestibular nucleus

MG : Medial geniculate body

MM : Medial division of medial mammillary nucleus

MP : Medial pontine nucleus

$\mathrm{mt}$ : Fasciculus mamillothalamicus

MV : Medial vestibular nucleus

PC . Nucleus of posterior commissure

PCO : Nucleus pontis centralis oralis

PH : Prepositus hypoglossi nucleus

PM : Premammillary nucleus

PO : Preoptic area

PV : Nucleus paraventricularis thalami

$\mathrm{R} \quad$ : Red nucleus

$\mathrm{RD}$ : Dorsal raphe nucleus

RP : Rostroventral pontine nucleus

RR : Retrorubral nucleus

RT : Nucleus reticularis tegmenti pontis

S : Subthalamic nucleus

SC : Superior colliculus

SE : Septal area

SG : Supragenual nucleus

SN : Substantia nigra

tc : Tractus corticospinalis

TD : Dorsal tegmental nucleus of Gudden

TDD : Pars dorsalis of TD

TDV : Pars ventralis of TD

TV : Ventral tegmental nucleus of Gudden

TVP : Pars principalis of TV

TZ : Nucleus of trapezoid body

Z : Zona incerta

3 : Oculomotor nucleus

4 : Trochlear nucleus

4VG : Gray matter along floor of fourth ventricle

5 : Nucleus motorius nervi trigemini

$5 \mathrm{M}$ : Nucleus mesencephalicus nervi trigemini

$5 \mathrm{~S}$ : Nucleus spinalis nervi trigemini

6N : Abducens nerve

$7 \mathrm{G}$ : Genu of facial nerve 


\section{PLATES}




\section{Explanation of Figures}

\section{Plate I}

Fig. 5. Brightfield photomicrographs of injection sites. A, Large WGA-HRP injection site into the RT in case G134. B. HRP injection site into the medial part of the RT in case G234. C. HRP injection site in to the lateral part of the RT in case G232.

Fig. 6. Brightfield photomicrograph of numerous WGA-HRP-labeled cells in the medial division of the medial mammillary nucleus (MM) after WGA-HRP injection into the RT in case G134.

Fig. 7. Darkfield photomicrograph of WGA-HRP-labeled cells in the medial division of the medial mammillary nucleus at the same section as in the Fig. 6 . Scale bar: $100 \mu \mathrm{m}$.

Fig. 8. Darkfield photomicrograph of HRP-labeled cells in the habenular complex in case G209. Arrows indicate HRP-labeled cells in the lateral edge of the medial habenular nucleus (MH), and the lateral habenular nucleus (LH). Scale bar: $100 \mu \mathrm{m}$. 

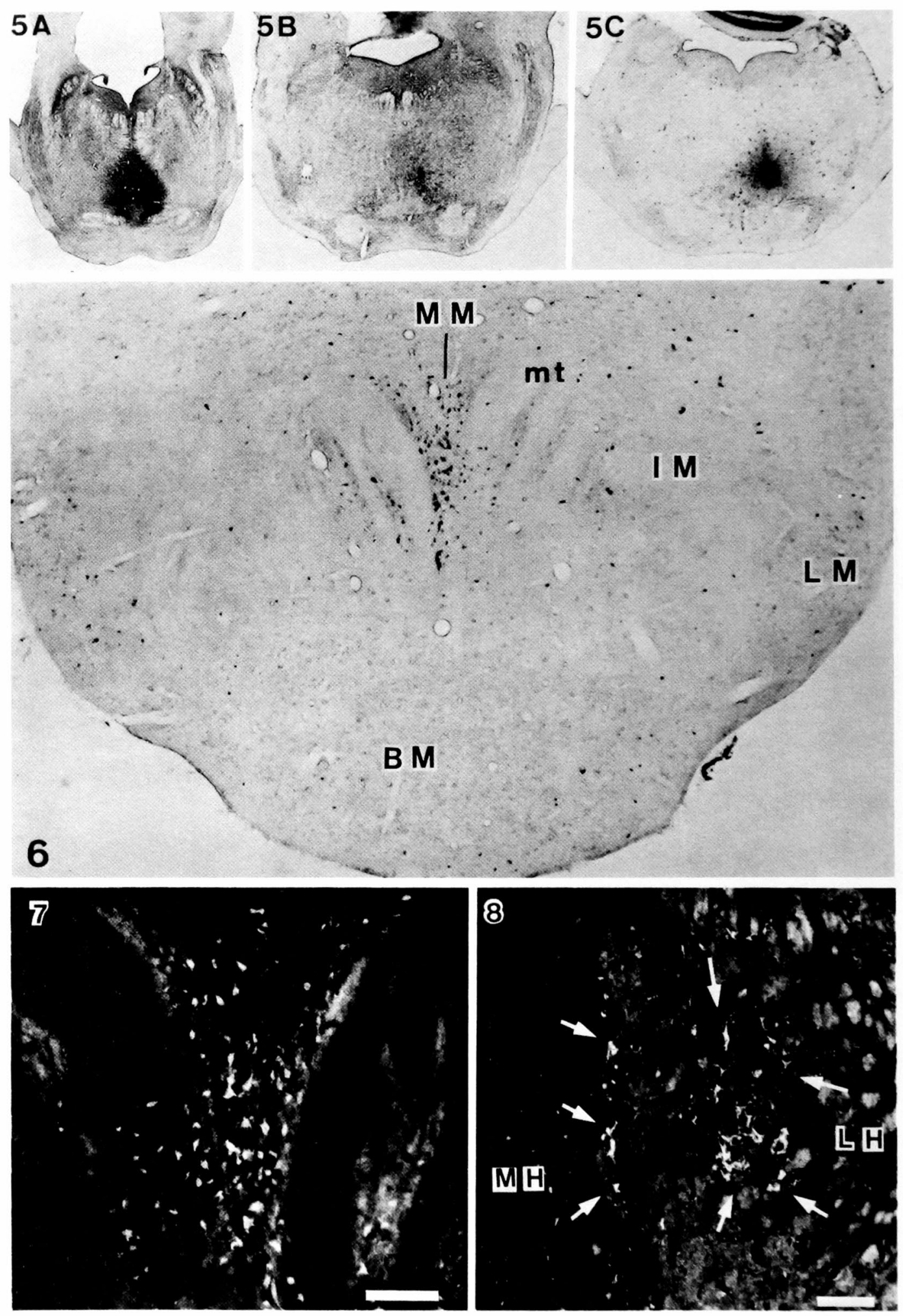


\section{Plate II}

Fig. 9. Brightfield photomicrograph of many HRP-lebeled cells in the ipsilateral nucleus of the fields of Forel after injection into the RT in case G134. Scale bar: $100 \mu \mathrm{m}$.

Fig. 10. Darkfield photomicrograph of HRP-labeled cells in the posterior nucleus of the interpeduncular complex after injection into the medial part of the RT in case G234. Arrows indicate HRP-labeled cells. Scale bar: $100 \mu \mathrm{m}$.

Fig. 11. Darkfield photomicrograph of HRP-labeled cells in the contralateral retrorubral nucleus after injection into the lateral part of the RT in case G232. Arrows indicate HRP-labeled cells. Scale bar: $100 \mu \mathrm{m}$.

Fig. 12. Brightfield photomicrograph of HRP-labeled cells in the ipsilateral nucleus prepositus hypoglossi after injection into the lateral part of the RT in case G232. Arrows indicate HRP-labeled cells. Scale bar: $100 \mu \mathrm{m}$.

Fig. 13. Brightfield photomicrograph of HRP-labeled cells in the contralateral supragenual nucleus after injection into the lateral part of the RT in case G232. Scale bar: $50 \mu \mathrm{m}$. 


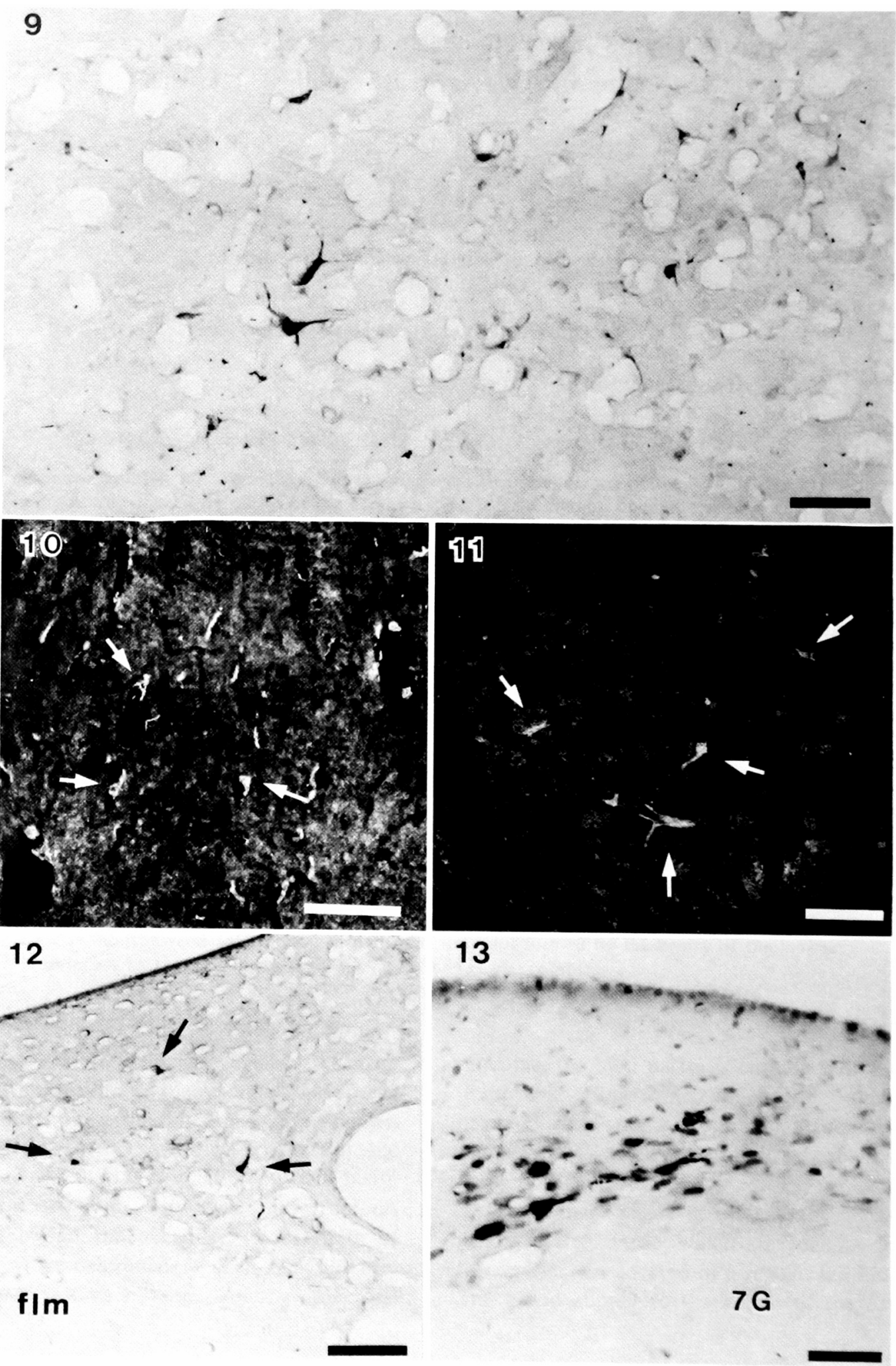

